\title{
Optical qubit by conditional interferometry
}

\author{
Matteo G. A. Paris * \\ Optics Section, Blackett Laboratory, Imperial College, London SW7 2BZ, United Kingdom \\ Dipartimento 'A. Volta' and Unitá INFM, Universitá di Pavia, via Bassi 6, I-27100 Pavia, Italy
}

(September 12, 2018)

\begin{abstract}
We suggest a method to prepare any chosen superposition $a_{0}|0\rangle+a_{1}|1\rangle$ of the vacuum and one-photon states. The method is based on a conditional double-interferometer fed by an one-photon state and a coherent state. The scheme involves only linear optical elements and avalanche photodetectors, and therefore it should be realizable with current technology. A realistic description of the triggering photodetectors is employed, i.e. we assume that they can only check, with a certain efficiency, whether or not any photon is present. We discuss two working regimes, and show that output states with fidelity arbitrarily close to unit may be obtained, with non vanishing conditional probability, also for low quantum efficiency at the photodetectors.
\end{abstract}

\section{INTRODUCTION}

The last two decades have witnessed a substantial development in quantum engineering and measurement of light. Several kinds of nonclassical states of light can now be generated, and their quantum properties can be fully characterized by accessible measurement schemes [1.2]. Besides fundamental interest, nonclassical states also find applications, as for exam-

\footnotetext{
${ }^{*}$ E-mail address:Matteo.Paris@pv.infn.it
} 
ple, the use of number states in quantum communication channels, and of squeezed light in high-precision gyroscopes and interferometers. More recently, the quantum engineering of light received new attention, which is mainly motivated by the potential improvement offered by quantum mechanics to the manipulation and the transmission of information [3]. Indeed, phenomena like teleportation [4], and quantum dense coding [5] found their first implementation in the quantum optical domain.

Photons do not interact, and this feature is very useful for the transmission of information without signal degradation. Indeed, the typical figures for losses in optical fibers are below $0.3 \mathrm{~dB} / \mathrm{Km}$. On the other hand, the same characteristic poses limitations to the manipulation of the quantum information encoded into a quantum state of light. Photonphoton interactions needed for computation, in fact, take place only in active optical media, characterized by nonlinear susceptibility. Usually, such nonlinearities are small, or masked by the concurrent absorption processes. Only recently, new methods based on dark atomic resonance and electromagnetically induced transparency [6] have been suggested to strongly enhance nonlinearity while suppressing absorption. The possibility of such giant nonlinearities renewed the interest for optical quantum technology, as it opens new perspectives to build single-photon quantum logic gates.

In this paper, we devote our attention to the preparation of any chosen superposition of the vacuum and the one-photons states $a_{0}|0\rangle+a_{1}|1\rangle$. This is the simplest state of light that carries a complete phase information, and, in turn, it represents the simplest example of an optical qubit. Remarkably, this is a low-energy-expense encoding of quantum information, as it requires, in average, less than one photon for each qubit. In particular, for the conventional computational basis $\left|\psi_{ \pm}\right\rangle=\frac{1}{\sqrt{2}}[|0\rangle \pm|1\rangle]$, i.e. for balanced superposition, half a photon for each qubit is required.

Different methods have been discussed with the purpose of engineering superpositions of radiation states. Mostly, these are in the context of cavity QED, since the interaction with atoms passing through the cavity allows to select specific components of an initial signal [7]. More recently, an all-optical device, based a ring cavity coupled to the signal through a Kerr- 
medium, has been suggested to realize Fock filtering, and thus preparation of superpositions [8]. In addition, a conditional scheme based on beam splitters and photocounters has been suggested to implement the optical state-truncation [9] of a coherent state, which, in turn, is used to prepare superpositions. As we will see, this last setup corresponds to a particular case of the present proposal.

The present scheme involves only linear optical elements and avalanche photodetectors, and therefore it should be realizable with current technology. In essence, it consists of two Mach-Zehnder interferometers arranged such that one of the outputs from the first one is then used as input for the second one. The first MZ is fed by an one-photon state, whereas the second is fed by a weak coherent state. The output states of the second interferometers are then measured, and the conditional output state from the first MZ turns out to be a superposition of the vacuum and one-photon states. The amplitudes for the two components can be tuned by varying the internal phase-shifts of the two interferometers and the amplitude of the coherent input.

The paper is structured as follows. In the next Section the scheme is presented and its dynamics is evaluated. Then, the ideal conditional output state is calculated for the case of a perfect photodetection process. In Section 11 w we take into account the imperfections of realistic photodetectors, and study their effects on the preparation of the superposition. In the literature two models of photodetectors (PDs) have been employed. In the first, which we use throughout the paper, it is assumed that PDs are only able to check, with a certain efficiency, whether or not photons are present. This is a reliable description of customary avalanche photodetectors, and we refer to this as YES/NO photodetection. In the second model, PDs are still affected by non unit quantum efficiency, but now they are able to discriminate among the number of incoming photons, thus acting as photocounters. This description does not yet correspond to available PDs, as no evidence of detectors capable to discriminate between the presence of, say, $n$ and $n+1$ photons for a generic $n$, have been reported. The photocounter model has been sometimes used in the literature on conditional measurements, remarkably in Ref. [9], and this led to the conclusion that 
schemes are reasonably insensitive to the detectors' inefficiency. In general, this is no longer true when the realistic features of avalanche photodetectors are taken into account. On the other hand, we will show that the present scheme offers a working regime in which the use of YES/NO detectors is enough to assure the reliable preparation of any chosen superposition. Finally, Section $[\nabla$ closes the paper with some concluding remarks.

\section{CONDITIONAL DOUBLE-INTERFEROMETRY}

The scheme we have in mind is the conditional double-interferometer depicted in Fig.

11. It consists of two Mach-Zehnder interferometers in cascade, in a way that makes one of the output signals from the first one to constitute one of the input signals for the second one. The three field-mode involved in the setup are denoted by $a, b$, and $c$, whereas the $B S$ 's are symmetric beam splitters. We also assume that equal and opposite phase-shifts, denoted by $\theta_{1}$ and $\theta_{2}$ respectively, are imposed in the arms of each interferometer (see Fig. 11). We assume that two interferometers are built with identical balanced beam splitters, and this means that they are fully characterized by the value of the internal phase-shift between their arms. The first interferometer is fed by an one-photon state in the mode $b$, whereas the other port, corresponding to mode $a$, is left unexcited. After this first stage the photon has a nonzero amplitude of being in both the output paths, whereas the values of such amplitudes are determined by the internal phase-shift $\theta_{1}$. The output mode $b$ is then mixed with mode $c$, prepared in a weak coherent state $|\gamma\rangle$, and they are both detected at the output of the second interferometer. Depending on the result of the measurements we have different conditional output states for the output mode $a$ of the first interferometer. In particular, we will see that any chosen superposition $a_{0}|0\rangle+a_{1}|1\rangle$ of the vacuum and one-photon states can be prepared with nonzero conditional probability.

The evolution operator of each interferometer can be written as

$$
\hat{V}_{M Z}(\theta)=\hat{V}_{B S} \exp \left\{i \theta\left(a^{\dagger} a-b^{\dagger} b\right)\right\} \hat{V}_{B S}^{\dagger}
$$

where 


$$
\hat{V}_{B S}=\exp \left\{i \frac{\pi}{4}\left(a^{\dagger} b+b^{\dagger} a\right)\right\}
$$

denotes the evolution operator of a symmetric beam splitter. Eq. (1) can be written as

$$
\hat{V}_{M Z}(\phi)=\exp \left\{i \frac{\pi}{2} b^{\dagger} b\right\} \exp \left\{-i \phi\left(a^{\dagger} b+b^{\dagger} a\right)\right\} \exp \left\{-i \frac{\pi}{2} b^{\dagger} b\right\}
$$

which shows that a Mach-Zehnder interferometer is equivalent to a single beam splitter $B S_{\phi}$ of transmissivity $\tau=\sin ^{2} \phi$, where $\phi=\theta / 2$, preceded and followed by rotations of $\pi / 2$ performed on one of the two modes [10]. After straightforward algebra, one may write the evolution operator of the whole device as

$$
\hat{U}\left(\phi_{1}, \phi_{2}\right)=\exp \left\{i \frac{\pi}{2} b^{\dagger} b\right\} \exp \left\{i \phi_{2}\left(b^{\dagger} c+c^{\dagger} b\right)\right\} \exp \left\{i \phi_{1}\left(a^{\dagger} b+b^{\dagger} a\right)\right\} \exp \left\{-i \frac{\pi}{2} b^{\dagger} b\right\}
$$

The overall input state can be written as

$$
\left|\Psi_{\mathrm{IN}}\right\rangle=|0\rangle_{a}|1\rangle_{b}|\gamma\rangle_{b}
$$

whereas using Eq. (4) we obtain the expression for the overall output

$$
\begin{aligned}
\left|\Psi_{\mathrm{OUT}}\right\rangle=\hat{U}\left(\phi_{1}, \phi_{2}\right)\left|\Psi_{\mathrm{IN}}\right\rangle= & \cos \phi_{1}|1\rangle_{a}\left|\gamma \cos \phi_{2}\right\rangle_{b}\left|\gamma \sin \phi_{2}\right\rangle_{c} \\
& +\sin \phi_{1} \sin \phi_{2}|0\rangle_{a} b^{\dagger}\left|\gamma \cos \phi_{2}\right\rangle_{b}\left|\gamma \sin \phi_{2}\right\rangle_{c} \\
& -\sin \phi_{1} \cos \phi_{2}|0\rangle_{a}\left|\gamma \cos \phi_{2}\right\rangle_{b} c^{\dagger}\left|\gamma \sin \phi_{2}\right\rangle_{c}
\end{aligned}
$$

We are now ready to analyze the effect on the mode $a$ of a measurement performed on the output modes $b$ and $c$. For the moment let us assume that the generic measurement of a quantity $X$ on the $b$ mode, and $Y$ on the $c$ mode is performed; we also assume that the two quantities are independent on each other. The measurement is thus described by a factorized probability operator measure $(\mathrm{POM}) \hat{\Pi}=\hat{\Pi}_{x} \otimes \hat{\Pi}_{y}$, where $x$ and $y$ denote the possible outcomes for the two quantities. The POMs are positive (hence selfadjoint) operators, if $\mathbb{X}$ and $\mathbb{Y}$ denote the spaces of the possible outcomes the normalization conditions can be written as ( $\widehat{\mathbb{I}}$ denotes the identity operator)

$$
\int_{\mathbb{X}} d x \hat{\Pi}_{x}=\widehat{\mathbb{I}} \quad \int_{\mathbb{Y}} d y \hat{\Pi}_{y}=\widehat{\mathbb{I}}
$$


The probability of the event $(x, y)$ is given by the global trace over the three modes

$$
P_{x y}=\operatorname{Tr}_{a b c}\left[\left|\Psi_{\text {oUT }}\right\rangle\left\langle\Psi_{\text {oUT }}\right| \hat{\Pi}_{x} \otimes \hat{\Pi}_{y}\right],
$$

whereas the corresponding conditional output state for the mode $a$ is given by the partial trace

$$
\hat{\varrho}_{x y}=\frac{1}{P_{x y}} \operatorname{Tr}_{b c}\left[\left|\Psi_{\text {oUT }}\right\rangle\left\langle\Psi_{\text {oUT }}\right| \hat{\Pi}_{x} \otimes \hat{\Pi}_{y}\right] .
$$

Actually, different measurements on $b$ and $c$ lead to different conditional output states, and since some events are more likely to occur than other, so are the corresponding conditional output states. In the following we consider the measurement of the photon number, and in particular we focus our attention to the case of a single photon recorded in one of the output modes, and no photons recorded in the other one. In order to establish notation we assume that the photon is recorded in the output mode $b$. However, the results are valid also for the case of of a photon registered in the mode $c$, up to a $\pi / 2$ shift in the internal phase-shifts of the second interferometer.

The ideal measurement of the photon number on the two output modes is described by the POM

$$
\hat{\Pi}_{n k}=|n\rangle_{b b}\langle n|\otimes| k\rangle_{c c}\langle k| \quad n, k=0,1, \ldots .
$$

Therefore, the detection probability for the case $n \equiv 1, k \equiv 0$ is equal to

$$
\left.P_{10}=\left|\left\langle\Psi_{\text {oUT }} \mid 1\right\rangle_{b}\right| 0\right\rangle\left._{c}\right|^{2}=e^{-|\gamma|^{2}}\left[\sin ^{2} \phi_{1} \sin ^{2} \phi_{2}+|\gamma|^{2} \cos ^{2} \phi_{1} \cos ^{2} \phi_{2}\right],
$$

and the conditional output state for the mode $a$ is given by

$$
\begin{aligned}
\hat{\varrho}_{10} & =\frac{1}{P_{10}} \operatorname{Tr}_{b c}\left[\left|\Psi_{\text {OUT }}\right\rangle\left\langle\Psi_{\text {OUT }}|| 1\right\rangle_{b b}\langle 1|\otimes| 0\rangle_{c c}\langle 0|\right]= \\
& =\frac{1}{P_{10}}\left[c_{00}|0\rangle\left\langle 0\left|+c_{11}\right| 1\right\rangle\left\langle 1\left|+c_{01}\right| 0\right\rangle\left\langle 1\left|+c_{10}\right| 1\right\rangle\langle 0|\right],
\end{aligned}
$$

where 


$$
\begin{aligned}
& c_{00}=e^{-|\gamma|^{2}} \sin ^{2} \phi_{1} \sin ^{2} \phi_{2} \quad c_{11}=e^{-|\gamma|^{2}}|\gamma|^{2} \cos ^{2} \phi_{1} \cos ^{2} \phi_{2} \\
& c_{01}=e^{-|\gamma|^{2}} \gamma \sin \phi_{1} \sin \phi_{2} \cos \phi_{1} \cos \phi_{2} \quad c_{10}=c_{01}^{*}
\end{aligned}
$$

the star denoting complex conjugation. By looking at Eqs. (13) it is easy to recognize that $\hat{\varrho}_{10}$ is actually a pure state $\hat{\varrho}_{10}=\left|\psi_{10}\right\rangle\left\langle\psi_{10}\right|$ where

$$
\left|\psi_{10}\right\rangle=\frac{\sin \phi_{1} \sin \phi_{2}|0\rangle+\gamma \cos \phi_{1} \cos \phi_{2}|1\rangle}{\sqrt{\sin ^{2} \phi_{1} \sin ^{2} \phi_{2}+|\gamma|^{2} \cos ^{2} \phi_{1} \cos ^{2} \phi_{2}}} .
$$

By varying the interferometric shifts, and the amplitude $\gamma$ of the coherent input $c$, we may achieve any chosen superposition of the vacuum and the one-photon states. In particular, the internal phase-shifts $\phi_{1}$ and $\phi_{2}$, and the modulus $|\gamma|$, govern the weights of the two component, whereas the relative phase equals the argument of the complex amplitude $\gamma$.

We notice that the truncation scheme of Ref. [9] is equivalent to a particular case of our setup, corresponding to the balanced choice $\phi_{1}=\phi_{2}=\pi / 4$. This is due, as mentioned above, to the fact that a Mach-Zehnder interferometer is substantially equivalent to a single beam splitter of transmissivity $\tau=\sin ^{2} \phi$. However, besides the fact that the present scheme offers additional degrees of freedom, the use of an interferometric setup has the specific advantage of a larger stability.

Remarkably, the superposition of Eq. (14) may be obtained by different values of $\phi_{1}$, $\phi_{2}$ and $\gamma$, and this degree of freedom can be used to maximize the corresponding detection probability $P_{10}$, i.e. the probability of the event which leaves the state of the mode $a$ into the desired superposition. Let us consider, for example, the preparation of a generic balanced superposition

$$
\left|\psi_{\star}\right\rangle=\frac{|0\rangle+e^{i \varphi}|1\rangle}{\sqrt{2}}
$$

In order to reduce Eq. (14) to Eq. (15) we need $\varphi=\arg \gamma$ and $|\gamma|=\tan \phi_{1} \tan \phi_{2}$. The detection probability is then given by

$$
P_{10}^{\star}=2 \sin ^{2} \phi_{1} \sin ^{2} \phi_{2} \exp \left[-\tan \phi_{1}^{2} \tan \phi_{2}^{2}\right]
$$

From Eq. (16) we have that $0<P_{10}^{\star} \lesssim 0.21$, with the maximum value reached for $\phi_{1} \equiv \phi_{2} \simeq$ 0.715, corresponding to an optimum amplitude $|\gamma|_{\text {opt }} \simeq 0.755$. However, the dependence 
of the detection probability on $\phi_{1}$ and $\phi_{2}$ is not dramatic, such that there exists a sizeable region in which the detection probability is above $P_{10}^{\star}>20 \%$ (see Fig. 2).

As mentioned above, the symmetric case of a photon detected in the output mode $c$ and no photons in $b$ leads to an equivalent result, up to the replacement $\phi_{2} \rightarrow \phi_{2}+\pi / 2$. In formula we have

$$
P_{01}=e^{-|\gamma|^{2}}\left[\sin ^{2} \phi_{1} \cos ^{2} \phi_{2}+|\gamma|^{2} \cos ^{2} \phi_{1} \sin ^{2} \phi_{2}\right]
$$

and

$$
\left|\psi_{01}\right\rangle=\frac{\sin \phi_{1} \cos \phi_{2}|0\rangle-\gamma \cos \phi_{1} \sin \phi_{2}|1\rangle}{\sqrt{\sin ^{2} \phi_{1} \cos ^{2} \phi_{2}+|\gamma|^{2} \cos ^{2} \phi_{1} \sin ^{2} \phi_{2}}} .
$$

By comparing Eqs. (14) and (18) we also note that the scalar product between the two conditional output state is given by

$$
\left|\left\langle\psi_{01} \mid \psi_{10}\right\rangle\right|^{2} \propto \sin \phi_{2} \cos \phi_{2}\left(\sin ^{2} \phi_{1}-|\gamma|^{2} \cos ^{2} \phi_{1}\right)
$$

which means that for $|\gamma|=\tan \phi_{1}$, and independently on $\phi_{2}$, the two states are orthogonal (this also happens for $\phi_{2}=p \pi / 2, p \in \mathbb{Z}$ and any $\gamma$ and $\phi_{1}$, but this case just corresponds to have one state in the vacuum and the other in the one-photon state). In this regime, the scheme provides a reliable source (i.e. with $P_{10}=P_{01}=\exp \left\{-\tan \phi_{1}^{2}\right\} \sin ^{2} \phi_{1}$ which is larger than $20 \%$ in the region around $\phi_{1} \simeq 0.67$ ) of a quantum-optical computational basis.

Of course, it is now of interest to study whether or not the superpositions of Eqs. (14) and (18) may be obtained in a realistic implementation of the setup. Since the scheme is based on conditional measurements, the main concern should be with the photodetection

process. Therefore, in the next Section we are going to take into account the imperfections of available photodetectors, in order to check the robustness of the preparation scheme against the detectors inefficiency.

\section{EFFECTS OF REALISTIC PHOTODETECTION}

Light is revealed by exploiting the interaction with atoms or molecules. Each photon ionizes a single atom, and the resulting charge is then amplified to produce a measurable 
pulse. In practice, however, available photodetectors are hardly performing the ideal measurement of the photon number. Their performances, in fact, are limited by two main kinds of imperfections. On one hand, photodetectors are usually characterized by a quantum efficiency lower than unit, which means that only a fraction of the incoming photons lead to an electric pulse, and ultimately to a "count". Some photons are either reflected from the surface of the detector, or are absorbed without being transformed into electric pulses. On the other hand, customary photodetectors involve an avalanche process to transform a single ionization event into a recordable pulse. This implies that it is very difficult to discriminate between the presence of a single photon or more than one.

The outcomes from such a detector may be either YES, which means a "click", corresponding to any number of photons, or NO, which means that no photons have been recorded. This kind measurement is described by a two-value POM

$$
\hat{\Pi}_{\mathrm{N}}=\sum_{p=0}^{\infty}(1-\eta)^{p}|p\rangle\langle p| \quad \hat{\Pi}_{\mathrm{Y}}=\widehat{\mathbb{I}}-\hat{\Pi}_{\mathrm{N}},
$$

where $\eta$ is the quantum efficiency, and $\widehat{\mathbb{I}}$ denotes the identity operator. Indeed, for high quantum efficiency (close to unit) $\hat{\Pi}_{\mathrm{N}}$ approaches the projection operator onto the vacuum state, and $\hat{\Pi}_{Y}$ onto the orthogonal subspace.

The event of observing a click at the PD surveying the output mode $b$ (i.e. $D_{b}$, see Fig. (1), and no photons at $D_{c}$, is characterized by the probability

$$
\begin{aligned}
P_{\mathrm{YN}}\left[\eta, \gamma, \phi_{1}, \phi_{2}\right] & =\operatorname{Tr}_{a b c}\left[\left|\Psi_{\text {OUT }}\right\rangle\left\langle\Psi_{\text {OUT }}\right| \hat{\Pi}_{\mathrm{Y}} \otimes \hat{\Pi}_{\mathrm{N}}\right]= \\
& =e^{-\eta|\gamma|^{2} \sin ^{2} \phi_{2}}\left\{1-e^{-\eta|\gamma|^{2} \cos ^{2} \phi_{2}}+\eta \sin ^{2} \phi_{1}\left[e^{-\eta|\gamma|^{2} \cos ^{2} \phi_{2}}\right.\right. \\
& \left.\left.+\cos ^{2} \phi_{2}\left(\eta|\gamma|^{2} \sin ^{2} \phi_{2}-1\right)\right]\right\} .
\end{aligned}
$$

The corresponding conditional output state is

$$
\begin{aligned}
\hat{\varrho}_{\mathrm{YN}} & =\frac{1}{P_{\mathrm{YN}}} \operatorname{Tr}_{b c}\left[\left|\Psi_{\text {OUT }}\right\rangle\left\langle\Psi_{\text {OUT }}\right| \hat{\Pi}_{\mathrm{Y}} \otimes \hat{\Pi}_{\mathrm{N}}\right]= \\
& =\frac{1}{P_{\mathrm{YN}}}\left[d_{00}|0\rangle\left\langle 0\left|+d_{11}\right| 1\right\rangle\left\langle 1\left|+d_{01}\right| 0\right\rangle\left\langle 1\left|+d_{01}^{*}\right| 1\right\rangle\langle 0|\right],
\end{aligned}
$$


where the coefficients are given by (see appendix A)

$$
\begin{aligned}
& d_{11}=e^{-\eta|\gamma|^{2} \sin ^{2} \phi_{2}} \cos ^{2} \phi_{1}\left[1-e^{-\eta|\gamma|^{2} \cos ^{2} \phi_{2}}\right] \\
& d_{00}=e^{-\eta|\gamma|^{2} \sin ^{2} \phi_{2}} \sin ^{2} \phi_{1}\left[1-(1-\eta) e^{-\eta|\gamma|^{2} \cos ^{2} \phi_{2}}+\eta \cos ^{2} \phi_{2}\left(\eta|\gamma|^{2} \sin ^{2} \phi_{2}-1\right)\right] \\
& d_{01}=e^{-\eta|\gamma|^{2} \sin ^{2} \phi_{2}} \eta \gamma \sin \phi_{1} \sin \phi_{2} \cos \phi_{1} \cos \phi_{2} .
\end{aligned}
$$

In general, the conditional output state $\hat{\varrho}_{\mathrm{YN}}$ is no longer a pure state. However, as we will see, there are regimes in which $\hat{\varrho}_{\mathrm{YN}}$ approaches the desired superposition. In order to compare $\hat{\varrho}_{\mathrm{YN}}$ with the ideal conditional output $\left|\psi_{10}\right\rangle$ we consider the fidelity $F=\left\langle\psi_{10}\left|\hat{\varrho}_{\mathrm{YN}}\right| \psi_{10}\right\rangle$. From Eqs. (6) and (22-23) we have

$$
\begin{aligned}
F\left[\eta, \gamma, \phi_{1}, \phi_{2}\right]= & \frac{1}{P_{\mathrm{YN}}} \frac{e^{-\eta|\gamma|^{2} \sin ^{2} \phi_{2}}}{\sin ^{2} \phi_{1} \sin ^{2} \phi_{2}+|\gamma|^{2} \cos ^{2} \phi_{1} \cos ^{2} \phi_{2}}\left\{|\gamma|^{2} \cos ^{4} \phi_{1} \sin ^{2} \phi_{2}\left(1-e^{-\eta|\gamma|^{2} \cos \phi_{2}^{2}}\right)\right. \\
& +2 \eta|\gamma|^{2} \sin ^{2} \phi_{1} \sin ^{2} \phi_{2} \cos ^{2} \phi_{1} \cos ^{2} \phi_{2}+\sin ^{4} \phi_{1} \sin ^{2} \phi_{2}\left[1-(1-\eta) e^{-\eta|\gamma|^{2} \cos ^{2} \phi_{2}}\right. \\
& \left.\left.+\eta \cos ^{2} \phi_{2}\left(\eta|\gamma|^{2} \sin ^{2} \phi_{2}-1\right)\right]\right\}
\end{aligned}
$$

Our goal is now to find regimes in which the fidelity of the conditional output state is close to unit and, at the same time, the corresponding detection probability $P_{\mathrm{YN}}$ does not vanish. In particular, we are interested in the preparation of those superpositions where the amplitudes of the two components are of the same order, thus assuring that the state is far from being just the vacuum or the one-photon state. This requirement roughly corresponds to the condition

$$
\sin \phi_{1} \sin \phi_{2} \simeq \cos \phi_{1} \cos \phi_{2}
$$

whereas a fine tuning of the amplitude, as well as the phase of the superposition, may be obtained by varying the complex amplitude $\gamma$ of the coherent input. The condition in Eq. (25) is satisfied by two different working regimes of the setup, i. e. by two different pairs of values of the internal phase-shifts. These are the balanced choice $\phi_{1}=\phi_{2}=\pi / 4$ and the unbalanced one $\phi_{1} \simeq 0 \phi_{2} \simeq \pi / 2-\phi_{1}$ respectively. In both cases, the general expression for the fidelity in Eq. (24) may be considerably simplified, and the corresponding working regime discussed with some details. 


\section{A. The case $\phi_{1}=\phi_{2}=\pi / 4$}

In the case $\phi_{1}=\phi_{2}=\pi / 4$, the detection probability rewrites as

$$
P_{\mathrm{YN}}[\eta, \gamma, \pi / 4, \pi / 4]=e^{-\frac{1}{2} \eta|\gamma|^{2}}\left\{1-e^{-\frac{1}{2} \eta|\gamma|^{2}}+\frac{1}{2} \eta\left[e^{\frac{1}{2} \eta|\gamma|^{2}}+\frac{1}{4}\left(\eta|\gamma|^{2}-2\right)\right]\right\},
$$

and the fidelity

$$
F[\eta, \gamma, \pi / 4, \pi / 4]=\frac{\left[4-2 \eta+|\gamma|^{2}(2+\eta)^{2}\right]-4 e^{-\frac{1}{2} \eta|\gamma|^{2}}\left(1-\eta+|\gamma|^{2}\right)}{\left(1+|\gamma|^{2}\right)\left[8+\eta\left(\eta|\gamma|^{2}-2\right)-4 e^{-\eta|\gamma|^{2} / 2}(2-\eta)\right]} .
$$

The detection probability shows relatively large values $\left(P_{\mathrm{YN}} \gtrsim 50 \%\right)$ in a sizeable region (see Fig. 3) of the $\eta-|\gamma|$ space, including also situations with low quantum efficiency. Unfortunately, the fidelity of Eq. (27) is a rapidly decreasing function of the coherent amplitude and, in the relevant region $0<|\gamma|^{2} \lesssim 4$, it is bounded by

$$
F_{\text {MAX }}<\frac{2+|\gamma|^{2}\left(9-4 e^{-|\gamma|^{2} / 2}\right)}{\left(1+|\gamma|^{2}\right)\left(|\gamma|^{2}+6-4 e^{-|\gamma|^{2} / 2}\right)} .
$$

This working regime is thus effective only for $|\gamma| \ll 1$, corresponding to the preparation of superposition where the vacuum component is preponderant. Indeed, for balanced superpositions $(|\gamma| \simeq 1)$ we have the bound $F \lesssim 93 \%$. As we will see in the following, this limitation can be overcome by the unbalanced tuning of the internal phase-shifts.

Our analysis of the balanced scheme led to conclusions that are in contrast with those of Ref. [9], where, as mentioned in Section [1], a formally equivalent scheme has been used. The reason for this disagreement stays in the different models employed to describe the photodetection process (see Appendix B). Actually, as far as we know, the YES/NO model used here is more realistic that the photocounter model used there, as, in fact, no evidence of detectors capable to discriminate between the number of incoming photons have been reported. We should conclude that the authors' hope of "reasonable insensitivity" to the detectors inefficiency [9] is not yet realized with current technology.

\section{B. The case $\phi_{1} \simeq 0, \phi_{2} \simeq \pi / 2-\phi_{1}$}

In the case $\phi_{1} \simeq 0, \phi_{2} \simeq \pi / 2-\phi_{1}$, the detection probability is given by 


$$
\begin{aligned}
P_{\mathrm{YN}}\left[\eta, \gamma, \phi_{1} \simeq 0, \phi_{2} \simeq \pi / 2-\phi_{1}\right] & \simeq e^{-\eta|\gamma|^{2}}\left[\eta|\gamma|^{2} \cos ^{2} \phi_{2}+\eta \sin ^{2} \phi_{1}\right] \\
& \simeq \eta \phi_{1}^{2}\left(1+|\gamma|^{2}\right) \exp \left[-\eta|\gamma|^{2}\right]
\end{aligned}
$$

and the fidelity

$$
F\left[\eta, \gamma, \phi_{1} \simeq 0, \phi_{2} \simeq \pi / 2-\phi_{1}\right] \simeq 1-\frac{1}{2} \phi_{1}^{2}(2-\eta)
$$

Remarkably, the fidelity is now independent on the amplitude of the coherent input, and can be made arbitrarily close to unit by choosing a smaller value for $\phi_{1}$. The price to pay for this result is a lower value of the detection probability, namely a lower efficiency of the preparation scheme. However, the resulting probability is still large enough to make the scheme an effective source of superposition states. As an example, let us consider $\phi_{1}$ such that $\sin ^{2} \phi_{1}=\cos ^{2} \phi_{2} \simeq 0.01$. In this case, we obtain a very high value of the fidelity $F>99 \%$, and yet a detection probability given by $P_{\mathrm{YN}} \simeq 1 \%$ (almost independently on the quantum efficiency). More generally, we can substitute Eq. (29) in Eq. (30) to write

$$
F \simeq 1-\frac{2-\eta}{2 \eta\left(1+|\gamma|^{2}\right)} e^{\eta|\gamma|^{2}} P_{\mathrm{YN}}
$$

Eqs. (29), (30) and (31) assure that a reliable generation of the desired superposition is achievable (with non vanishing probability) also for low quantum efficiency at photodetectors.

\section{Balanced superpositions}

We end the Section by illustrating the performance of the setup in preparing the special class of exactly balanced superpositions of Eq. (15). The requirement for equal amplitudes reads $|\gamma|=\tan \phi_{1} \tan \phi_{2}$. By substitution in Eqs. (21) and (22) we obtain the detection probability and the corresponding conditional output state. We do not show here the resulting expressions, which are rather cumbersome. Instead, in Fig. 4 , we report the behavior of both, the fidelity and the detection probability, as a function of the internal phase-shifts for two values of the quantum efficiency at the photodetectors. As it is apparent from the plots, 
there always exists a region, in which the fidelity is very close to unit, and yet the detection probability is larger than $10 \%$. Therefore, for balanced superpositions, the performances with realistic detectors do not substantially differ from that obtained in the ideal working regime discussed in Section 11 .

\section{CONCLUSIONS}

We have analyzed a linear, conditional, interferometric setup to prepare any chosen superposition $a_{0}|0\rangle+a_{1}|1\rangle$ of the vacuum and one-photon states. It consists of a three-port double-interferometer fed by a one-photon state and a coherent state. The scheme involves only linear optical elements and avalanche photodetectors, and therefore should be of interest from the point of view of the experimental realization. In principle, i. e. in case of a perfect photodetection process, the setup can be used to generate any chosen superposition with a conditional probability about 20\%. The imperfections of realistic photodetectors have been taken into account, and their effects have analyzed in details. An optimal working regime has been found, in which output states arbitrarily close to the desired superposition is obtained with non vanishing conditional probability. Typical values for the fidelity are above $F \geq 99 \%$, with conditional probability about $P \simeq 1 \%$. For the relevant case of balanced superposition, the detection probability may be increased by a fine tuning of the amplitude of the coherent input. In this case, the performances of the setup are approaching the ideal working regime.

\section{ACKNOWLEDGMENTS}

This work has been cosponsored by C.N.R. and N.A.T.O. through the Advanced Fel-

lowship Program 1998. The author thanks Peter Knight and Martin Plenio for their kind hospitality at Imperial College. 


\section{REFERENCES}

[1] See, for example, the special issues: J. Mod. Opt. 44 (1997), on "Quantum state preparation and measurement", and Acta Phys. Slov. 48 (1998) on "Quantum Optics and Quantum Information".

[2] D.-G Welsch, W. Vogel, T. Opatrny, Prog. Opt., 39 (1999).

[3] C. H. Bennett, Phys. Scr. T76, 210 (1998).

[4] D. Boschi, S. Branca, F. De Martini, L. Hardy, S. Popescu, Phys. Rev. Lett. 80, 1121 (1998); S. L. Braunstein, H. J. Kimble, Phys. Rev. Lett. 80, 869 (1998).

[5] C. H. Bennett, S. J. Wiesner, Phys. Rev. Lett. 69, 2881 (1992); K. Mattle, H. Weinfurter, P. G. Kwiat, A. Zeilinger, Phys. Rev. Lett. 76, 1895 (1996).

[6] H. Schmidt and A. Imamoglu, Opt. Lett. 21, 1936 (1996); L. V. Hau, S. E. Harris, Z. Dutton, and C. H. Behroozi, Nature 397, 594 (1999); S. Rebić, S. M. Tan, A. S. Parkins, D. F. Walls, J. Opt. B1,490 (1999).

[7] J. Krause, M. O. Scully, T. Walther, and H. Walther, Phys. Rev. A 39, 1915 (1989); M. Kozierowski, and S. M. Chumakov, Phys. Rev. A 52, 4194 (1995);P. Domokos, M. Brune, J. Raimond, S. Haroche, Eur. Phys. J. D 1, 1 (1998).

[8] G. M. D’Ariano, L. Maccone, M. G. A. Paris, M. F. Sacchi, Acta Phys. Slov. 49, 659 (1999); see also quant-ph 9906077.

[9] D. T. Pegg, L. S. Philips, S. M. Barnett, Phys. Rev. Lett. 81, 1604 (1998).

[10] M. G. A. Paris, Phys. Rev. A 59, 1615 (1999).

\section{APPENDIX A: CONDITIONAL DENSITY MATRIX FOR REALISTIC PHOTODETECTION}

Starting from Eqs. (6), (8) and (20) we have 


$$
\begin{aligned}
d_{11} & =\cos ^{2} \phi_{1}\left\langle\beta\left|\hat{\Pi}_{\mathrm{Y}}\right| \beta\right\rangle\left\langle\delta\left|\hat{\Pi}_{\mathrm{N}}\right| \delta\right\rangle \\
d_{00} & =\sin ^{2} \phi_{1}\left[\sin ^{2} \phi_{2}\left\langle\beta\left|b \hat{\Pi}_{\mathrm{Y}} b^{\dagger}\right| \beta\right\rangle\left\langle\delta\left|\hat{\Pi}_{\mathrm{N}}\right| \delta\right\rangle+\cos ^{2} \phi_{2}\left\langle\beta\left|\hat{\Pi}_{\mathrm{Y}}\right| \beta\right\rangle\left\langle\delta\left|c \hat{\Pi}_{\mathrm{N}} c^{\dagger}\right| \delta\right\rangle\right. \\
& \left.-\sin \phi_{2} \cos \phi_{2}\left(\left\langle\beta\left|\hat{\Pi}_{\mathrm{Y}} b^{\dagger}\right| \beta\right\rangle\left\langle\delta\left|c \hat{\Pi}_{\mathrm{N}}\right| \delta\right\rangle+\left\langle\beta\left|b \hat{\Pi}_{\mathrm{Y}}\right| \beta\right\rangle\left\langle\delta\left|\hat{\Pi}_{\mathrm{N}} c^{\dagger}\right| \delta\right\rangle\right)\right] \\
d_{01} & =\sin \phi_{1} \cos \phi_{1}\left(\sin \phi_{2}\left\langle\beta\left|\hat{\Pi}_{\mathrm{Y}} b^{\dagger}\right| \beta\right\rangle\left\langle\delta\left|\hat{\Pi}_{\mathrm{N}}\right| \delta\right\rangle+\cos \phi_{2}\left\langle\beta\left|\hat{\Pi}_{\mathrm{Y}}\right| \beta\right\rangle\left\langle\delta\left|\hat{\Pi}_{\mathrm{N}} c^{\dagger}\right| \delta\right\rangle\right)
\end{aligned}
$$

where $\beta=\gamma \cos \phi_{2}$ and $\delta=\gamma \sin \phi_{2}$. Let us denote by $|z\rangle$ a generic coherent state with

complex amplitude $z \in \mathbb{C}$, then by using the definition (20) of the $\operatorname{POM}\left\{\hat{\Pi}_{\mathrm{N}}, \hat{\Pi}_{\mathrm{Y}}\right\}$ we have

$$
\begin{aligned}
\left\langle z\left|\hat{\Pi}_{\mathrm{N}}\right| z\right\rangle & =\exp \left\{-\eta|z|^{2}\right\} \\
\left\langle z\left|a \hat{\Pi}_{\mathrm{N}}\right| z\right\rangle & =z(1-\eta) \exp \left\{-\eta|z|^{2}\right\} \\
\left\langle z\left|a \hat{\Pi}_{\mathrm{N}} a^{\dagger}\right| z\right\rangle & =(1-\eta)\left[1+|z|^{2}(1-\eta)\right] \exp \left\{-\eta|z|^{2}\right\},
\end{aligned}
$$

and

$$
\begin{aligned}
\left\langle z\left|\hat{\Pi}_{\mathrm{Y}}\right| z\right\rangle & =1-\left\langle z\left|\hat{\Pi}_{\mathrm{N}}\right| z\right\rangle \\
\left\langle z\left|a \hat{\Pi}_{\mathrm{Y}}\right| z\right\rangle & =z-\left\langle z\left|a \hat{\Pi}_{\mathrm{N}}\right| z\right\rangle \\
\left\langle z\left|a \hat{\Pi}_{\mathrm{Y}} a^{\dagger}\right| z\right\rangle & =1+|z|^{2}-\left\langle z\left|a \hat{\Pi}_{\mathrm{N}} a^{\dagger}\right| z\right\rangle .
\end{aligned}
$$

Eventually, upon inserting Eqs. (A2) and (A3) in Eq. (A1) we arrive at the expression (23) for the coefficients of the conditional output state.

\section{APPENDIX B: MODELING DETECTORS AS PHOTOCOUNTERS}

By modeling a detector as a photocounter we assume that it is able to discriminate among pulses of different amplitudes, ideally corresponding to the different number of recorded photons. Actually, the number of "clicks" cannot be not the number of incoming photons, as the photocounter is characterized by a nonunit quantum efficiency $\eta$. The POM describing the measurement is given by a Bernoulli convolution of the ideal photon-number POM $\hat{\Pi}_{n}=|n\rangle\langle n|$. In formula, we have 


$$
\hat{\Pi}_{n}^{\eta}=\sum_{k=n}^{\infty} \eta^{n}(1-\eta)^{k-n}\left(\begin{array}{l}
k \\
n
\end{array}\right)|k\rangle\langle k| .
$$

Therefore, compared to the picture of detectors as avalanche photodetectors, we have that the operator probability for the vacuum detection is the same, i.e. $\hat{\Pi}_{\mathrm{N}}=\hat{\Pi}_{0}^{\eta}$, whereas for the case of a single click

$$
\hat{\Pi}_{1}^{\eta}=\frac{\eta}{1-\eta} \sum_{k=1}^{\infty} k(1-\eta)^{k}|k\rangle\langle k| .
$$

Eqs. (A3) are now transformed into

$$
\begin{aligned}
\left\langle z\left|\hat{\Pi}_{1}^{\eta}\right| z\right\rangle & =\eta|z|^{2} e^{-\eta|z|^{2}} \\
\left\langle z\left|a \hat{\Pi}_{1}^{\eta}\right| z\right\rangle & =\eta|z|^{2} e^{-\eta|z|^{2}}\left[1+|z|^{2}(1-\eta)\right] \\
\left\langle z\left|a \hat{\Pi}_{1}^{\eta} a^{\dagger}\right| z\right\rangle & =\eta|z|^{2} e^{-\eta|z|^{2}}\left[1+3|z|^{2}(1-\eta)+|z|^{4}(1-\eta)\right],
\end{aligned}
$$

which leads to a detection probability given by

$$
P_{10}^{\eta}=\eta\left[\sin ^{2} \phi_{1} \sin ^{2} \phi_{2}+|\gamma|^{2} \cos ^{2} \phi_{2}\left(1-\eta \sin ^{2} \phi_{1}\right)\right],
$$

and to a conditional output state whose coefficients are expressed as

$$
\begin{aligned}
& d_{11}=\eta|\gamma|^{2} \cos ^{2} \phi_{1} \cos ^{2} \phi_{2} \\
& d_{00}=\eta \sin ^{2} \phi_{1}\left[\sin ^{2} \phi_{2}+|\gamma|^{2}(1-\eta) \cos ^{2} \phi_{2}\right] \\
& d_{01}=\eta \sin \phi_{1} \sin \phi_{2} \cos \phi_{1} \cos \phi_{2}\left[1+2|\gamma|^{2}(1-\eta) \cos ^{2} \phi_{2}\right] .
\end{aligned}
$$

For the balanced setting $\phi_{1}=\phi_{2}=\pi / 4$ we have $P_{10}^{\eta}=\eta / 4\left[1+|\gamma|^{2}(2-\eta)\right]$ and

$$
\begin{aligned}
& d_{11}=\frac{|\gamma|^{2}}{1+|\gamma|^{2}(2-\eta)} \\
& d_{00}=d_{01}=\frac{1+|\gamma|^{2}(1-\eta)}{1+|\gamma|^{2}(2-\eta)} .
\end{aligned}
$$

Finally, the fidelity is given by

$$
F[\eta, \gamma]=1-\frac{|\gamma|^{4}(1-\eta)}{\left(1+|\gamma|^{2}\right)\left[1+|\gamma|^{2}(2-\eta)\right]},
$$

which is the result reported in Ref. [9]. The fidelity of Eq. (B7) varies in the range $5 / 6 \leq$ $F \leq 1$ as a function of the quantum efficiency at the photodetectors. 


\section{FIGURES}

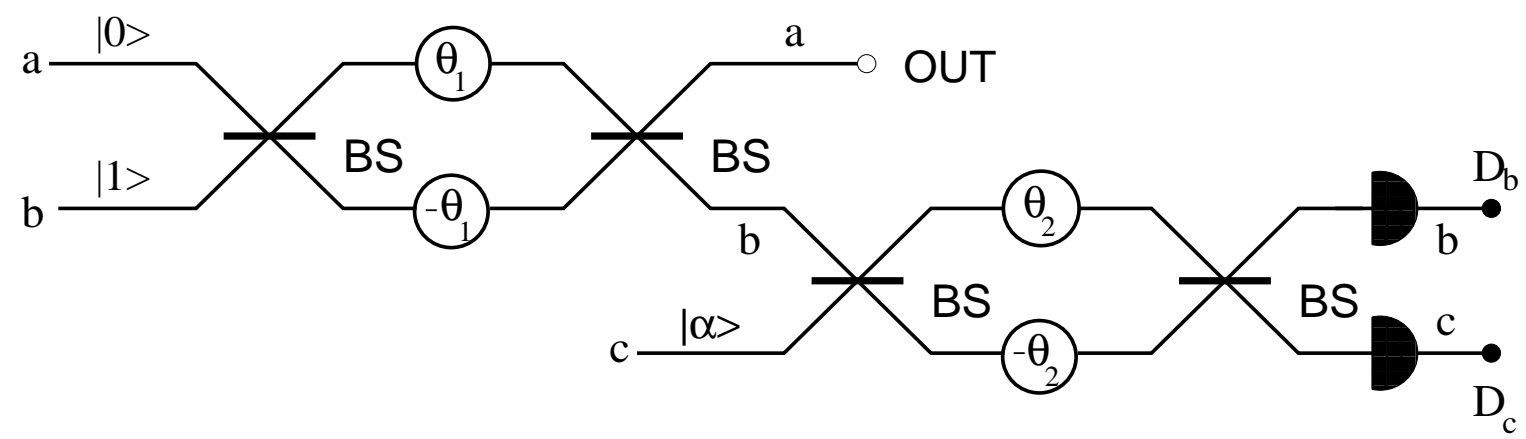

FIG. 1. Schematic diagram of the conditional interferometric setup for the preparation of any chosen superposition $a_{0}|0\rangle+a_{1}|1\rangle$ of the vacuum and one-photon states. The BS' are identical balanced beam splitters, whereas $D_{a}$ and $D_{c}$ denote two identical avalanche photodetectors. The first stage consists of a Mach-Zehnder interferometer fed by an one-photon state in mode $b$. Then, one of the output from the first interferometer is used as input of the other one, whose second port (mode $c$ ) is excited in a weak coherent state. Both the output modes from the second interferometer are detected by avalanche photodetectors, and depending on the observed result we obtain different conditional output state in the mode $a$ (denoted by OUT in the picture). The event of recording one photon in one of the photodetectors (either $D_{b}$ or $D_{c}$ ) and no photons in the other one corresponds to the preparation of a superposition of the vacuum and one-photon states. The relative weights of the two components may be tuned by varying either the internal phase-shifts $\theta_{1}$ and $\theta_{2}$ or the amplitude $|\gamma|$ of the coherent input, whereas the the phase of superposition equals the argument $\arg \gamma$ 


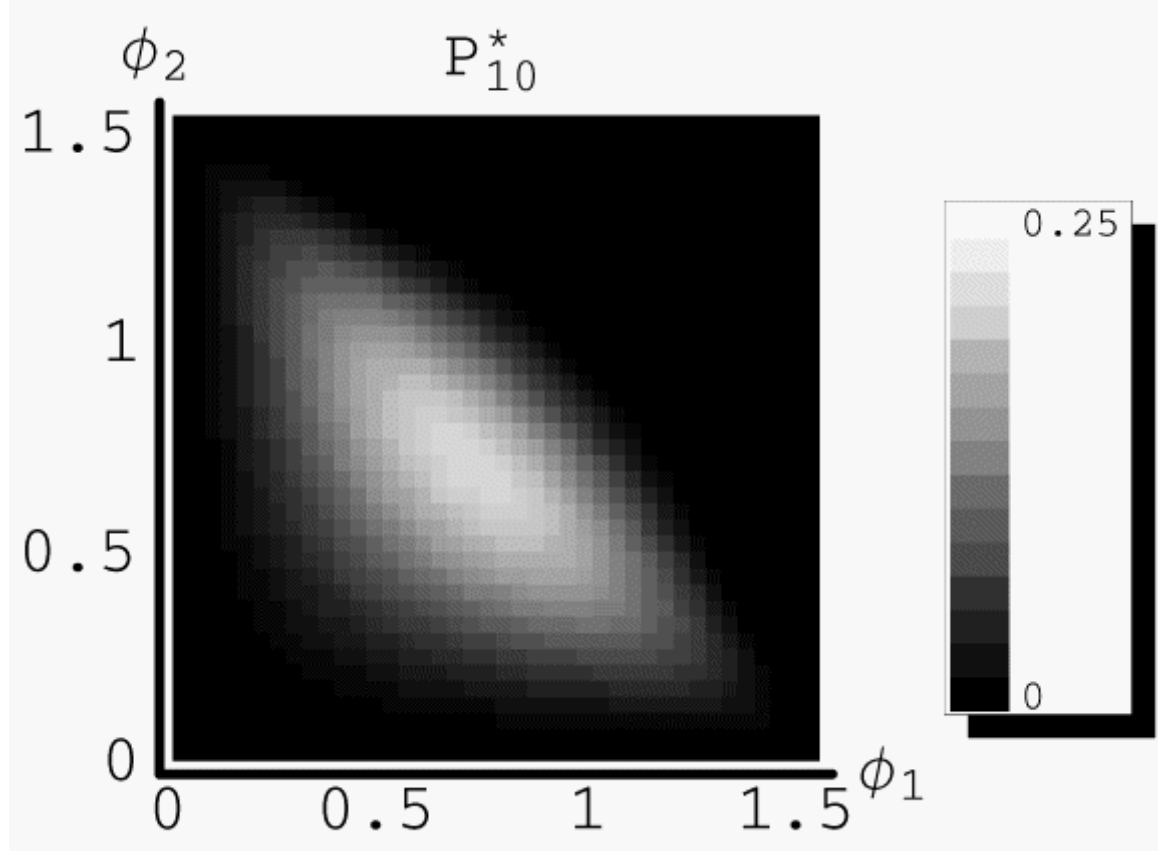

FIG. 2. Density plot of the conditional detection probability $P_{10}^{\star}$ for the preparation of the balanced superposition of Eq. (15). The maximum value $\left(P_{10}^{\star} \simeq 21 \%\right)$ is reached for $\phi_{1} \equiv \phi_{2} \simeq 0.715$, corresponding to an optimum amplitude $|\gamma|_{\text {opt }} \simeq 0.755$. However, the dependence of the detection probability on $\phi_{1}$ and $\phi_{2}$ is not dramatic, such, as it is apparent from the plot, there exists a sizeable region in which the detection probability is above $P_{10}^{\star}>20 \%$. 

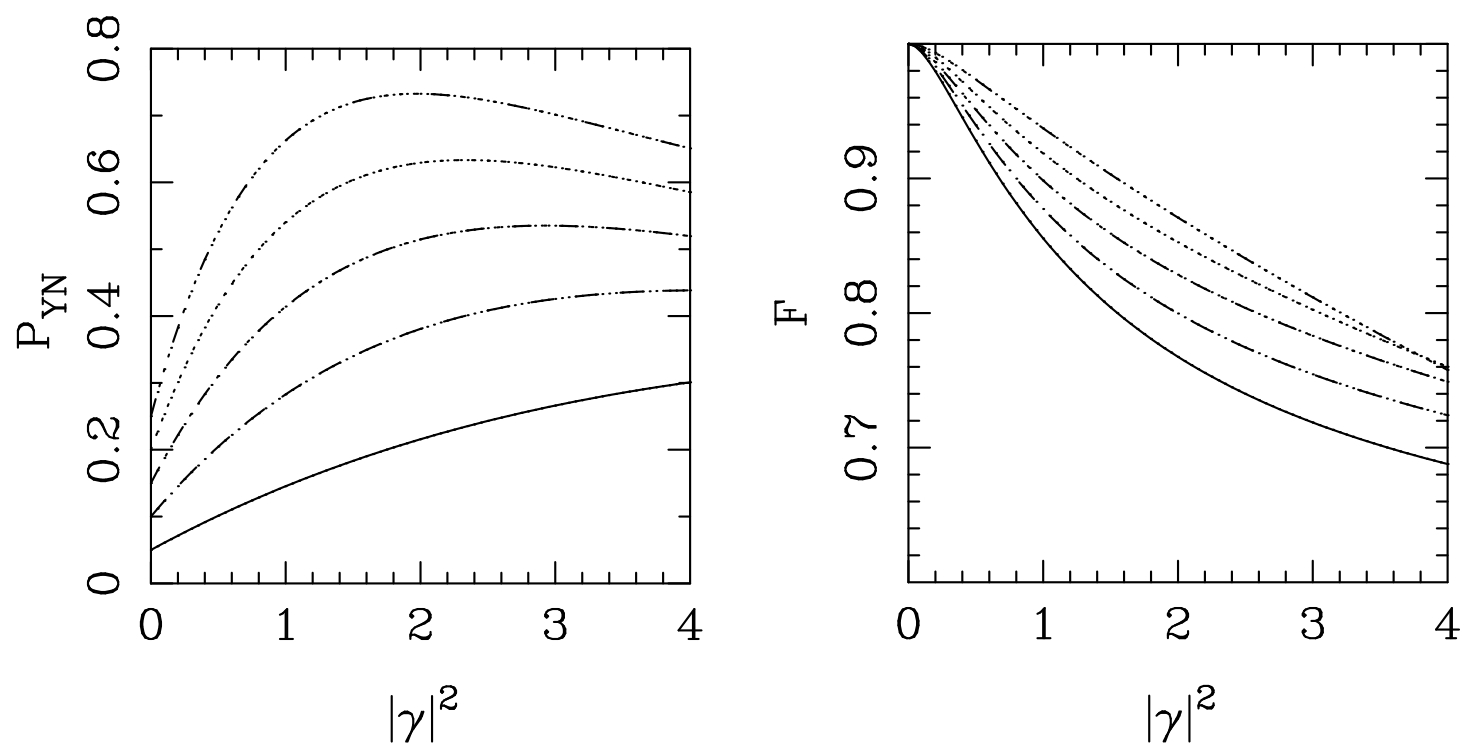

FIG. 3. Performances of the setup with realistic description of the photodetectors: the case of balanced choice for the internal phase-shifts. The figure shows the detection probability $P_{10}$ (on the left) and fidelity $F$ to the desired superposition (on the right) as a function of the intensity of the input coherent state for different values of the quantum efficiency of the photodetectors. In both plots we have, from bottom to top, $\eta=20 \%$ (solid line), $40 \%, 60 \%, 80 \%$, and $100 \%$.
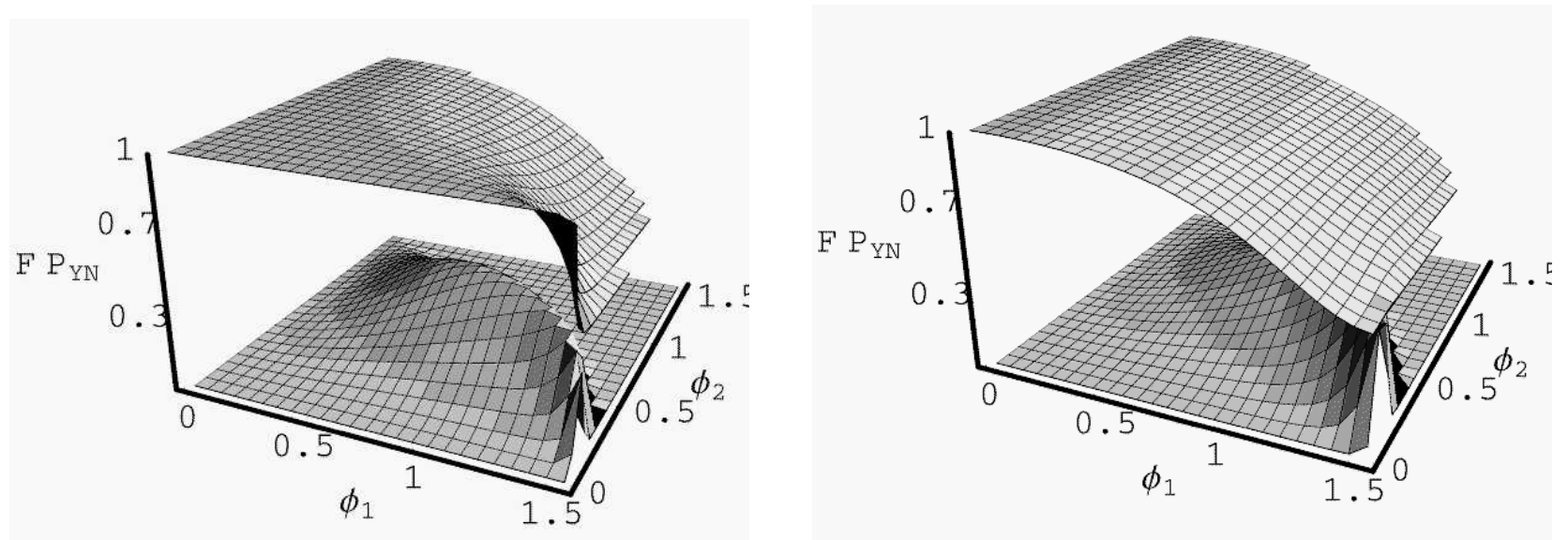

FIG. 4. Performances of the setup with realistic description of the photodetectors: preparation of balanced superpositions. The figure shows the detection probability $P_{10}$ and the fidelity $F$ to the desired superposition as a function of the internal phase-shifts $\phi_{1}$ and $\phi_{2}$ for unit quantum efficiency (on the left) and for $\eta=50 \%$ (on the right). 